\title{
Analysis of Nano-scale Strain Near Shallow Trench Isolation Structures by Energy-filtered Convergent Beam Electron Diffraction
}

\author{
Peng Zhang,,${ }^{* * *, * * *}$ John Mardinly, ${ }^{* * *}$ Oleh Karpenko, ${ }^{* * *}$ Andrei Istratov,,${ }^{* * *}$ Haifeng He, ${ }^{* *}$ Joel \\ Ager, ${ }^{* *}$ Chris Nelson, ${ }^{* *}$ Eric Stach, ${ }^{* * * *}$ Christian Kisielowski, ${ }^{* *}$ Eicke Weber ${ }^{*}{ }^{* *}$, and John Spence ${ }^{* *}$ \\ * Department of Materials Science and Engineering, University of California, Berkeley, CA 94720 \\ ** Materials Sciences Division, Lawrence Berkeley National Laboratory, Berkeley, CA 94720 \\ *** Technology Manufacturing Group, Intel Corporation, Santa Clara, CA 95054 \\ ${ }^{* * * *}$ School of Materials Engineering, Purdue University, West Lafayette, IN 47907
}

Shallow trench isolation (STI) is used in silicon technology for electrical isolation between devices. However, stress near the oxide-filled trenches may lead to defect generation and may affect device performance. A crucial task for the development of sub-100nm device technologies is to measure strain at nanometer spatial resolution and control it at nanoscale. Although the convergent beam electron diffraction (CBED) technique of transmission electron microscopy (TEM) has been successfully applied to measure highly localized strain around STI structures [1-3] where the intertrench silicon islands have sizes larger than 200nm, it is not clear whether CBED can be used to characterize local strain beyond the $90 \mathrm{~nm}$ technology node.

In this work we studied STI structures used for $90 \mathrm{~nm}$ and $130 \mathrm{~nm}$ flash memory device fabrication. To evaluate the strain-detection sensitivity of CBED, after identical trench lithography, different high density plasma (HDP) steps were used for trench oxide filling. The Zeiss Libra ${ }^{\circledR} 200 \mathrm{kV}$ TEM used in this study features a monochromator, an in-column OMEGA energy filter, and a high-angle annular dark-field detector, which make it ideal for spot-to-spot CBED measurement [4]. By using a $20 \mathrm{eV}$ energy selection slit, most plasmon peaks are excluded for CBED pattern formation. The $\chi^{2}$ fitting [5] of lattice parameters is implemented by the Automatic Strain Analysis by CBED (ASAC) package of Soft Imaging System. Use of the automated procedure has been discussed by Armigliato et al $[1,2]$. We have used the silicon [340] zone axis for these experiments.

An example of the cross-sectional TEM micrograph of the structures and the CBED patterns collected from sample\#1 is presented in Fig.1. As we can see from Fig.1(a)-(c), the HOLZ lines either split or become blurred in the small silicon island. The pattern quality is much better in the adjacent larger island as shown in Fig.1(d)-(f). Lattice parameters could be determined only from Fig.1(a),(d), and (e) which have acceptable quality. The fitted lattice parameters and $\chi^{2}$ values are listed in Table 1. It appears that the nitride cap provides tensile stress and partially compensates the compression from oxide fillings near the pad oxide.

For two other samples (\#2 and \#3) prepared with modified HDP recipes, we could get sufficiently sharp HOLZ patterns only near the top of the larger silicon island. However, at this position, one could not isolate the contribution from the isolation nitride. So, we collected patterns $15 \mathrm{~nm}$ off the bottom of the trench (corresponding to point 7 in Fig.1). For these two samples, it is difficult to tell the difference in strain from only one CBED measurement per sample due to significant noise. For accurate statistics, over ten patterns were collected at the identical position for different trenches in the same TEM sample. The averaged lattice parameters and standard deviation values are given in Table 2. It is found that the compressive strain in sample \#2 is larger than in \#3. 
The application of the CBED technique is limited by at least two characteristics which generate strain gradient along the path of electron beam and blur HOLZ lines: (a) a spontaneous elastic strain relaxation and local lattice distortion from thinning the TEM sample to electron-transparency, and (b) HOLZ lines are usually collected along low-symmetry axes [1, 2] which are several degrees off the [110] axis to minimize dynamical scattering effects. The blurring of CBED patterns is more pronounced in smaller devices due to higher stress gradients in such devices. In our presentation, we will discuss possible sample preparation techniques to minimize this effect.

References:

[1] A. Armigliato, R. Balboni, G. P. Carnevale, G. Pavia, D. Piccolo, S. Frabboni, A. Benedetti, and A. G. Cullis, Appl. Phys. Lett. 82 (2003) 2172.

[2] A. Armigliato, R. Balboni, and S. Frabboni, Appl. Phys. Lett. 86 (2005) 063508.

[3] M. Kim, J. M. Zuo, and G. S. Park, Appl. Phys. Lett. 84 (2004) 2181.

[4] J. C. H. Spence and J. M. Zuo, Electron Microdiffraction. New York, Plenum Press, 1992.

[5] J. M. Zuo, Ultramicroscopy 41 (1992) 211.

[6] UC Berkeley group is supported by SiWEDS. We acknowledge the use of the facilities of the National Center for Electron Microscopy, Lawrence Berkeley Lab, which is supported by the U.S. Department of Energy under Contract \#DE-AC02-05CH11231. We acknowledge the support of Department of Energy under Contract \#DOE. FG03-02ER45596. We thank Drs. A. Armigliato, M. Wibbelt, and J. Krieger for establishing the ASAC loaning license provided by Soft-Imaging Systems. The authors acknowledge the assistance provided by the TEM group and Thin Films group of Intel Corporation at Santa Clara, California.
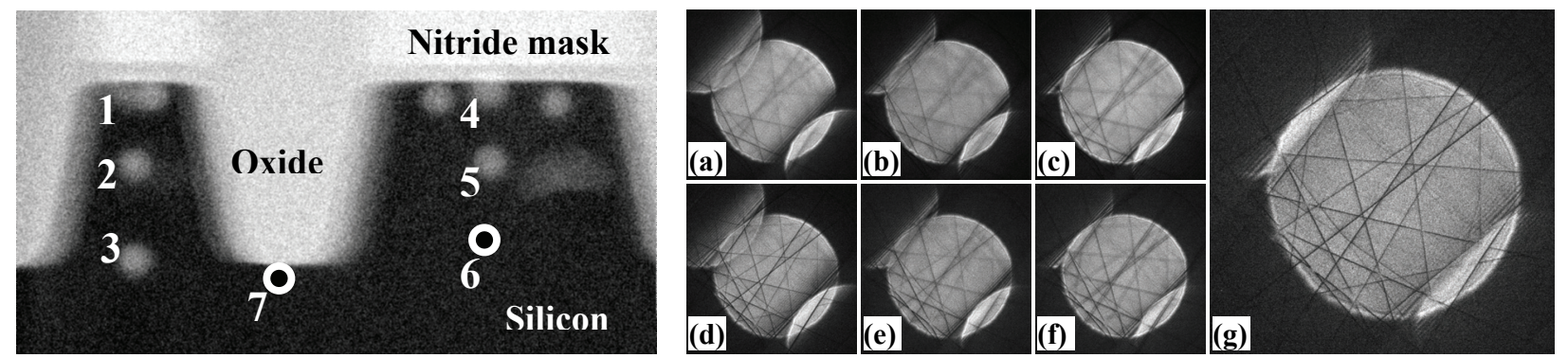

Fig. 1. On the left: Dark field STEM image of the STI structures taken along Si [340] zone axis. Contamination spots (white dots) were left on the sample after excessive e-beam exposure. On the right: CBED patterns (a)-(f) taken at points 1-6 depicted on the TEM image on the left, respectively.

(g) was taken from bulk silicon. No digital processing has been applied to these patterns.

Table 1. Lattice parameters measured at different positions (a), (d), and (e) of sample\#1.

\begin{tabular}{|c|c|c|c|c|c|c|c|c|}
\hline Pattern & $\mathrm{a}(\AA)$ & $\mathrm{b}(\AA)$ & $\mathrm{c}(\AA)$ & $\alpha$ (degree) & $\beta$ (degree) & $\gamma$ (degree) & $\chi^{2}$ & $\begin{array}{c}\text { Trace of strain } \\
\text { tensor }\left(\times 10^{-4}\right)\end{array}$ \\
\hline (a) & 5.4303 & 5.4303 & 5.4312 & 89.98 & 90.02 & 89.98 & 1.0396 & -2.2 \\
\hline$(\mathrm{d})$ & 5.4298 & 5.4298 & 5.4344 & 90.01 & 89.99 & 89.99 & 0.4907 & 1.8 \\
\hline$(\mathrm{e})$ & 5.4277 & 5.4277 & 5.4301 & 89.97 & 90.03 & 89.95 & 1.0243 & -13.8 \\
\hline
\end{tabular}

Table 2. Lattice parameters measured at $15 \mathrm{~nm}$ off the bottom of oxide filling for sample \#2 and \#3.

\begin{tabular}{|c|c|c|c|c|c|c|c|}
\hline Sample & $\mathrm{a}(\AA)$ & $\mathrm{b}(\AA)$ & $\mathrm{c}(\AA)$ & $\alpha$ (degree) & $\beta$ (degree) & $\gamma($ degree) & $\begin{array}{c}\text { Trace of strain } \\
\text { tensor }\left(\times 10^{-4}\right)\end{array}$ \\
\hline \multirow{2}{*}{$\# 2$} & 5.4310 & 5.4310 & 5.4283 & 90.00 & 90.00 & 90.00 & $-4.9 \pm 1.3$ \\
& \pm 0.0003 & \pm 0.0003 & \pm 0.0007 & \pm 0.01 & \pm 0.01 & \pm 0.02 & \\
\hline \multirow{2}{*}{$\# 3$} & 5.4312 & 5.4312 & 5.4299 & 90.00 & 90.00 & 90.00 & $-1.3 \pm 3.2$ \\
& \pm 0.0007 & \pm 0.0007 & \pm 0.0010 & \pm 0.02 & \pm 0.02 & \pm 0.01 & \\
\hline
\end{tabular}

